\title{
Interplay of Charge and Spin Coherence in Landau-Zener-Stückelberg-Majorana Interferometry
}

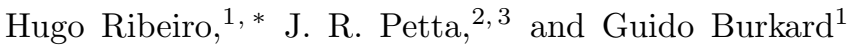 \\ ${ }^{1}$ Department of Physics, University of Konstanz, D-78457 Konstanz, Germany \\ ${ }^{2}$ Department of Physics, Princeton University, Princeton, New Jersey 08544, USA \\ ${ }^{3}$ Princeton Institute for the Science and Technology of Materials (PRISM), \\ Princeton University, Princeton, New Jersey 08544, USA
}

\begin{abstract}
We study Landau-Zener dynamics in a double quantum dot filled with two electrons, where the spin states can become correlated with charge states, and the level velocity can be tuned in a timedependent fashion. We show that a correct interpretation of experimental data is only possible when finite-time effects are taking into account. In addition, our formalism allows the study of partial adiabatic dynamics in the presence of phonon-mediated hyperfine relaxation and charge noise induced dephasing. Our findings demonstrate that charge noise severely impacts the visibility of LZSM interference fringes. This indicates that charge coherence must be treated on an equal footing with spin coherence.
\end{abstract}

\section{INTRODUCTION}

Electron spins trapped in quantum dots (QDs) are promising candidates for implementing a scalable quantum computer $\frac{112}{12}$. Most of the DiVincenzo criteria, which state physical requirements a system must fulfill to achieve quantum computing, have been achieved with spin qubits, including initialization ${ }^{3}$, readout ${ }^{4}$, and coherent contro ${ }^{5}-7$. While experimental progress has been impressive, many of these methods are not yet accurate enough to allow large scale quantum computing with single spins. A key challenge can be appreciated by considering the relevant timescales associated with the spin dynamics. In GaAs quantum dot devices, it is well known that the hyperfine interaction leads to a randomly fluctuating nuclear field, $B_{\mathrm{n}} \sim 2 \mathrm{mT}$, which results in a 10-20 ns inhomogeneous spin dephasing time ${ }^{[5|8| 9}$. For comparison, the Rabi period obtained in a GaAs double quantum dot (DQD) using conventional electron spin resonance (ESR) is on the order of $110 \mathrm{~ns}{ }^{6}$. The use of spin-orbit driven electric dipole spin resonance (EDSR) in GaAs leads to even slower Rabi periods of roughly 210 $n s^{7}$. Therefore, in the case of single spin rotations, gate operation times are nearly an order of magnitude slower than the inhomogeneous spin dephasing time. This issue is not specific to GaAs based nanostructures. In InAs nanowires, where the spin-orbit interaction is larger than in GaAs, a Rabi period of $\sim 17 \mathrm{~ns}{ }^{10}$ was reported. However, it still is twice as long as the spin dephasing time of $\sim 8 \mathrm{~ns}$.

Viewed from a different perspective, the maximum ac field generated in DQD ESR experiments is on the order of $2 \mathrm{mT}$, which is the same magnitude as the fluctuating nuclear field ${ }^{6}$. As a result, single spin rotations in GaAs qubits follow imperfect trajectories on the Bloch sphere, resulting in a reduced oscillation visibility and gate errors ${ }^{6}$. Single spin selectivity imposes an additional challenge upon the development of a spin-based quantum processor. Magnetic fields generated in ESR are difficult to localize on the nanometer scale. Without g-factor con- trol, or local magnetic field gradients, the spins located in a quantum register would rotate at the same rate in the presence of a global ESR field. The long term goal is to be able to drive selective single spin rotations, without affecting neighboring spins that are on average only 20-50 nm away.

Instead of using the spin-up and spin-down states of a single electron, the qubit basis states can be represented by two (out of four) two-electron spin states confined in a DQD 11. For a qubit whose basis states are encoded in the singlet $\mathrm{S}$ and triplet $\mathrm{T}_{0}$ spin states of a $\mathrm{DQD}$, the two-electron exchange interaction allows for fast singlequbit gates (hundreds of picoseconds) ${ }^{5}$. Recent experiments $\frac{12113}{}$ have also demonstrated the possibility of realizing a conditional two-qubit gate. Within this two-spin version of a qubit, the two-qubit gate realized in Ref. 5 can be interpreted as a single qubit operation. However the exchange interaction only allows for rotations about a single axis, whereas to generate arbitrary rotations one needs two perpendicular rotation axes. The generation of a nuclear magnetic field gradient ${ }^{14}$ provides rotations about a second, non-collinear axis. While remarkable, this method also presents some difficulties when it has to be extended to a large number of qubits. It requires that the nuclear polarization is controlled in each DQD to create the desired gradient field. An advantage of this method is that the generation of the nuclear field gradient reduces nuclear spin fluctuations, resulting in an increase in the spin dephasing time ${ }^{15}$.

Recently, it has been proposed to use a two-spin basis consisting of the singlet $\mathrm{S}$ and triplet $\mathrm{T}_{+}$spin states $16-18$. Quantum control of the $\mathrm{S}-\mathrm{T}_{+}$qubit relies on LandauZener-Stückelberg-Majorand ${ }^{19} 22$ (LZSM) physics, which occurs in the system when the $\mathrm{S}-\mathrm{T}_{+}$qubit is repeatedly swept through the hyperfine mediated $\mathrm{S}_{-} \mathrm{T}_{+}$anticrossing. This all-electrical method also has the advantage of addressing individually each quantum dot.

LZSM physics describing the passage of a two-level quantum system through an anti-crossing can be applied to different fields of physics and chemistry ${ }^{23}$. In quan- 
tum information science, LZSM theory describes accurately the observed interference fringes (Stückelberg oscillations) of a superconducting qubit driven back and forth through its anti-crossing 24 . The LZSM description also accurately describes the coherent manipulation of a two-spin qubit encoded in the $\mathrm{S}$ and triplet $\mathrm{T}_{+}$ spin states, with dynamics driven by repeated passages through a hyperfine-mediated anti-crossing 16[17. In selfassembled quantum dots, LZSM theory has been used to design high-fidelity all-optical control of spin-based qubits 25.27 .

In this article we develop a quantum master equation to describe partial adiabatic passages between the spin singlet $\mathrm{S}$ and triplet $\mathrm{T}_{+}$states in the presence of both the fluctuating Overhauser field and the fluctuating charge environment. With our theory, we show charge dynamics can significantly hinder LZSM interferometry of spin states. While most of the interesting spin dynamics happens in the $(1,1)$ charge configuration, with one electron per dot, initialization and measurements are done deep in the $(2,0)$ charge configuration, where both electrons are in the left dot. Here $(l, r)$ denotes the number of electrons in the left and right dot. Crossing the $(1,1) \leftrightarrow(2,0)$ interdot charge transition necessarily involves charge dynamics. Since superpositions of charge states dephase on shorter time scales than superpositions of spin states, it is essential to consider this fast effective decoherence mechanism when spin and charge degrees of freedom become correlated during spin qubit evolution $28 \mid 29$. In particular, we use our formalism to analyze spin-charge dynamics associated with detuning pulses that have a tunable level velocity, with a high level velocity away from the anticrossing and a slow level velocity in the vicinity of the anti-crossing 18 .

The paper is organized as follows. We start by reviewing standard Landau-Zener theory, which is valid for an infinitely long ramp through an anti-crossing with a constant level velocity. In Sec. II we review the solution of the finite-time LZSM model, which can be used to model realistic experiments, and we demonstrate within the scope of this theory how fine tuning of the level velocity can be used to increase the visibility of the quantum oscillations. Section III focuses on the physical implementation of LZSM physics in a two-electron spin qubit. We derive an effective Hamiltonian describing the dynamics of the states in the vicinity of the $\mathrm{S}_{-} \mathrm{T}_{+}$anti-crossing. Compared to previously derived effective Hamiltonians ${ }^{17}$, we include the effects of charge superposition states in the spin-dependent anti-crossing. The last part of the section is devoted to the derivation of a master equation that describes the evolution of the density matrix. In Sec. IV, we first compare solutions of the master equation obtained with exprimental pulse profiles and measurements performed on a GaAs double quantum dot. We then show theory results for the singlet return probability for which we explore the effects of charge induced decoherence.

\section{ADIABATIC CONTROL OF A QUANTUM TWO-LEVEL SYSTEM}

There are numerous problems in physics that deal with the physics of two-level systems. The most common example is Rabi's formula ${ }^{30}$ which describes the occupation of a two-level system that is driven by a coherent field. In quantum information science, Rabi oscillations are widely used, e.g., to manipulate an electron spin confined in a $\mathrm{QD}^{31}$. Another widely studied problem involving only two quantum levels is adiabatic passage, which is commonly employed in nuclear magnetic resonance 32 . The physics of adiabatic passage can be found in a variety of systems, and several theoretical models have been developed to describe different kinds of adiabatic pro$\operatorname{cesses}^{33} 42$. There is, however, a particular description that has proven to be applicable in many distinct fields of physics: the Landau-Zener $\underline{19 \sqrt{20}}$ model. We refer to it in this article as the LZSM model, since it was independently studied by Stückelberg 21 and Majorana ${ }^{22}$.

The Hamiltonian studied in the LZSM model describes a system with two energy levels [see Fig. 11(a)] that are coupled by an off-diagonal matrix element $\lambda, H(t)=$ $-(\alpha t / 2) \sigma_{z}+\lambda \sigma_{x}$, where $\sigma_{z}$ and $\sigma_{x}$ are Pauli matrices, and $\alpha=\mathrm{d}\left(E_{2}(t)-E_{1}(t) / \mathrm{d} t\right.$. The main result of the theory is the asymptotic expression for the non-adiabatic transition probability when the propagation lasts from $t_{\mathrm{i}}=-\infty$ to $t_{\mathrm{f}}=\infty$

$$
P_{\mathrm{LZSM}}=\mathrm{e}^{\frac{-2 \pi \lambda^{2}}{\alpha \hbar}} .
$$

Here we use a generalization of the LZSM model,

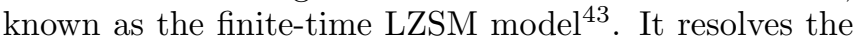
problem of the energy divergence when $t_{\mathrm{f}, \mathrm{i}}= \pm \infty$, and in contrast to the simple case it yields the relative phase between the states, which is crucial for predicting the coherent time evolution of any quantum system. Particularly, knowledge of the relative phase is essential in LZSM interferometry 2123 in which the system is driven back and forth across an anti-crossing. The driving generates quantum interference between states, which is directly observable in the non-adiabatic (or adiabatic) transition probability.

\section{A. Finite-time}

Landau-Zener-Stückelberg-Majorana propagator

The unitary evolution operator defined by the LZSM Hamiltonian 192 ,

$$
H(t)=\left(\begin{array}{cc}
-\frac{\alpha}{2} t & \lambda \\
\lambda & \frac{\alpha}{2} t
\end{array}\right)
$$

is given by 43

$$
U\left(t_{\mathrm{f}}, t_{\mathrm{i}}\right)=\left(\begin{array}{ll}
u_{11}\left(t_{\mathrm{f}}, t_{\mathrm{i}}\right) & u_{12}\left(t_{\mathrm{f}}, t_{\mathrm{i}}\right) \\
u_{21}\left(t_{\mathrm{f}}, t_{\mathrm{i}}\right) & u_{22}\left(t_{\mathrm{f}}, t_{\mathrm{i}}\right)
\end{array}\right),
$$




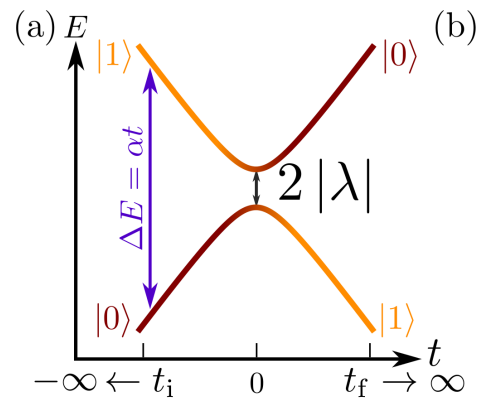

(c) $\Delta E$

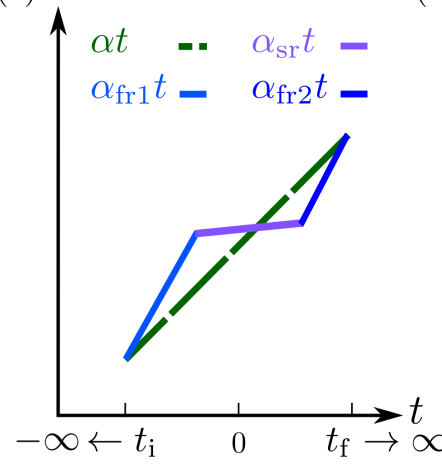

(d) $t$
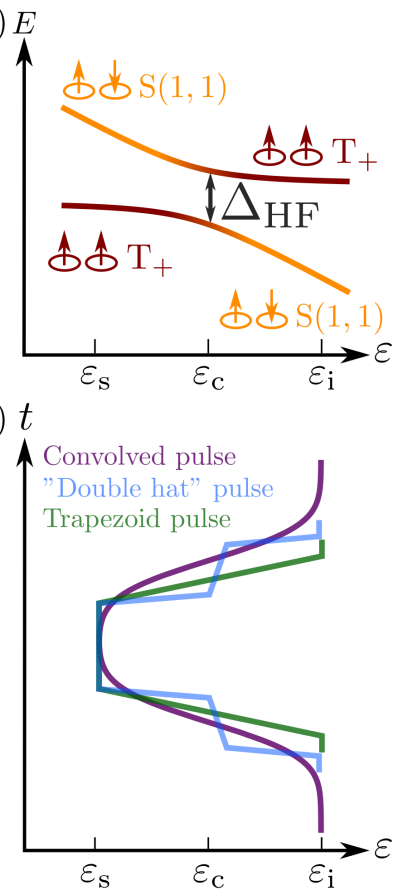

Figure 1. (color online) (a) The Landau-Zener-StückelbergMajorana (LZSM) problem. The LZSM model addresses the problem of a two-level system that is swept through an anticrossing. It assumes an infinitely long ramp (i.e. propagation from $t_{\mathrm{i}} \rightarrow-\infty$ to $t_{\mathrm{f}} \rightarrow \infty$ ), a constant coupling constant $\lambda$ leading to a splitting $2|\lambda|$ at $t=0$, and an energy difference between the levels that varies linearly with time, $\Delta E(t)=\alpha t$. Its main result gives the non-adiabatic transition probability, $P_{\text {LZSM }}$. An extension of the model to finite-times resolves the problem of infinite energies and undefined phases. (b) Singlet and triplet $\mathrm{T}_{+}$energies in a DQD as a function of detuning $\varepsilon$, where $\varepsilon_{\mathrm{i}}=\varepsilon\left(t_{\mathrm{i}}\right)$ and $\varepsilon_{\mathrm{s}}=\varepsilon\left(t_{\mathrm{f}}\right)$. The two-electron DQD is a physical realization of the LZSM model. Here, the hyperfine coupling of the two spin states leads to a splitting $\Delta_{\mathrm{HF}}$. (c) The LZSM model assumes a constant level velocity $\alpha$. In order to increase adiabaticity one can lower $\alpha$, but in order to keep short pulses it is preferable to use multi rise-time pulses. (d) Comparison of possible pulses that can be used to manipulate the $\mathrm{S}-\mathrm{T}_{+}$qubit. In terms of total propagation time, "double hat" pulses are a good compromise between conventional trapezoid and convolved pulses.

where $1(2)$ refers to the states $|0\rangle(|1\rangle)$ with

$$
\begin{aligned}
& u_{11}\left(t_{\mathrm{f}}, t_{\mathrm{i}}\right)=u_{22}^{*}\left(t_{\mathrm{f}}, t_{\mathrm{i}}\right)= \\
& \frac{\Gamma\left(1-\mathrm{i} \eta^{2}\right)}{\sqrt{2 \pi}}\left[D_{\mathrm{i} \eta^{2}}\left(\mathrm{e}^{\frac{-\mathrm{i} \pi}{4}} \tau_{\mathrm{f}}\right) D_{\mathrm{i} \eta^{2}-1}\left(\mathrm{e}^{\frac{3 \mathrm{i} \pi}{4}} \tau_{\mathrm{i}}\right)\right. \\
& \left.+D_{\mathrm{i} \eta^{2}}\left(\mathrm{e}^{\frac{3 \mathrm{i} \pi}{4}} \tau_{\mathrm{f}}\right) D_{\mathrm{i} \eta^{2}-1}\left(\mathrm{e}^{-\frac{\mathrm{i} \pi}{4}} \tau_{\mathrm{i}}\right)\right],
\end{aligned}
$$

and

$$
\begin{aligned}
& u_{12}\left(t_{\mathrm{f}}, t_{\mathrm{i}}\right)=-u_{21}^{*}\left(t_{\mathrm{f}}, t_{\mathrm{i}}\right)= \\
& \frac{\Gamma\left(1-\mathrm{i} \eta^{2}\right)}{\sqrt{2 \pi} \eta} \mathrm{e}^{\frac{\mathrm{i} \pi}{4}}\left[-D_{\mathrm{i} \eta^{2}}\left(\mathrm{e}^{\frac{-\mathrm{i} \pi}{4}} \tau_{\mathrm{f}}\right) D_{\mathrm{i} \eta^{2}}\left(\mathrm{e}^{\frac{3 \mathrm{i} \pi}{4}} \tau_{\mathrm{i}}\right)\right. \\
& \left.+D_{\mathrm{i} \eta^{2}}\left(\mathrm{e}^{\frac{3 \mathrm{i} \pi}{4}} \tau_{\mathrm{f}}\right) D_{\mathrm{i} \eta^{2}}\left(\mathrm{e}^{-\frac{\mathrm{i} \pi}{4}} \tau_{\mathrm{i}}\right)\right] .
\end{aligned}
$$

Here $\tau=\sqrt{\alpha / \hbar} t$ is a dimensionless time, $\eta=\lambda / \sqrt{\alpha \hbar}$ is a dimensionless coupling, $\Gamma(z)$ is the gamma function $\underline{44}$, and $D_{\nu}(z)$ is the parabolic cylinder function 45 . By definition, $t=0$ is set at the anti-crossing. The usual LZSM formula eq. (1) is retrieved from the modulus square of Eq. (4) when taking the limit $t_{\mathrm{i}} \rightarrow-\infty$ and $t_{\mathrm{f}} \rightarrow \infty$.

The LZSM propagator fully determines the partial adiabatic dynamics of a quantum two-level system. In the case where the two-level system encodes a qubit, Eq. (3) allows the design of single qubit operations. This method has been used to control superconducting qubits $23 \mid 24$, and more recently to implement a qubit encoded in the spin of a two-electron state $16 \mid 17$. However, since the spin states are weakly coupled, $\eta<1$, it is hard to achieve an equally weighted coherent superposition of spin states and fully explore the entire qubit state space. In order to achieve full control over the spin qubit, it would be necessary to perform slower sweeps, i.e., to increase $\eta$ by making $\alpha$ smaller. However, the LZSM equation requires an exponential increase in the propagation time in order to achieve a fully adiabatic transition. In a real physical system, this method is unpractical because the pulse duration needs to remain shorter than the coherence time of the two-level system.

\section{B. Observing finite-time effects}

As demonstrated in Ref. 18, it is possible to use more complex pulses to increase the balance of the populations while keeping the manipulation time below the decoherence times. The key idea relies on an observation based on the finite-time LZSM model. For a slow-level velocity $\alpha$, which favors adiabatic passage, most of the population change occurs in the vicinity of the anti-crossing. It is therefore possible to use detuning pulses that have a time-dependent level velocity. Let us consider two types of pulses, as illustrated in Figs. 1(c) and (d). The first is a conventional linear pulse, which is standard in LZSM theory. The second pulse profile consists of linear detuning ramps in a fast-slow-fast rise-time sequence, which we refer to as "double hat" pulse. The unitary evolution of such a general sequence can be written using Eq. (3) as

$$
\begin{aligned}
U\left(t_{\mathrm{f}}, t_{\mathrm{i}}\right) & =U_{\text {fast2 }}\left(t_{\mathrm{f}}, t_{2}\right) U_{\text {slow }}\left(t_{2}, t_{1}\right) U_{\text {fast } 1}\left(t_{1}, t_{\mathrm{i}}\right) \\
& =\left(\begin{array}{ll}
\tilde{u}_{11}\left(t_{\mathrm{f}}, t_{\mathrm{i}}\right) & \tilde{u}_{12}\left(t_{\mathrm{f}}, t_{\mathrm{i}}\right) \\
\tilde{u}_{21}\left(t_{\mathrm{f}}, t_{\mathrm{i}}\right) & \tilde{u}_{22}\left(t_{\mathrm{f}}, t_{\mathrm{i}}\right)
\end{array}\right) .
\end{aligned}
$$

Here $t_{1}=t_{\mathrm{i}}+t_{\mathrm{fr} 1}, t_{2}=t_{\mathrm{i}}+t_{\mathrm{fr} 1}+t_{\mathrm{sr}}$, and $t_{\mathrm{f}}=$ $t_{\mathrm{i}}+t_{\mathrm{fr} 1}+t_{\mathrm{sr}}+t_{\mathrm{fr} 2}$, where $t_{\mathrm{fr} j}$ is the propagation time associated to the $j$ th fast sequence, and $t_{\mathrm{sr}}$ corresponds to the slow sequence. We use this notation to refer to the corresponding level velocities $\alpha_{j}$, dimensionless times $\tau_{j}$, and dimensionless couplings $\eta_{j}$.

In addition to the already mentioned and studied advantages "double hat" pulses offer, they also provide sensitive means to explore finite-time effects. These are 
in general neglected when describing experiments because the more convenient LZSM scattering approach 23 has been sufficient to reproduce experimental results $\$ 16124$. However, to implement high-fidelity quantum gates it will be necessary to accurately describe the dynamics of the qubit and thus take into account finite-time propagation.

In Fig. 2 we compare adiabatic transition probabilities obtained with "double hat" pulses and conventional trapezoid pulses [c.f. inset of Fig. 2]. Here, an adiabatic transition refers to a transition where the system remains in an instantaneous energy eigenstate. The details of the leading edge (the trailing edge is identical but reversed) of the "double hat" pulse are $t_{\text {fr } 1}=t_{\text {fr2 }}=0.1 \mathrm{~ns}$, the starting position of the slow-level velocity ramp is defined by the condition $\Delta E_{\mathrm{i}}-\Delta E_{\mathrm{i}, \mathrm{sr}}=-2 \mu \mathrm{eV}$, where $\Delta E_{\mathrm{i}}$ is the initial energy difference between the uncoupled eigenstates and $\Delta E_{\mathrm{i}, \mathrm{sr}}$ the energy difference at the beginning of the slow part of the pulse. We choose $\alpha_{\mathrm{sr}}=500 \mathrm{eVs}^{-1}$ and $\lambda=70 \mathrm{neV}$. We impose the same total propagation time on the linear pulse as for the "double hat", from which we obtain the level velocity $\alpha_{\mathrm{si}}$ for the linear pulse,

$$
\alpha_{\mathrm{si}}=\frac{\Delta E_{\mathrm{f}}-\Delta E_{\mathrm{i}}}{t_{\mathrm{fr} 1}+t_{\mathrm{fr} 2}+t_{\mathrm{sr}}} .
$$

The adiabatic transition probability, $P_{\mathrm{a}}$, is plotted as a function of $\Delta E_{\mathrm{f}}$. Here, $\Delta E_{\mathrm{f}}$ indicates the energy difference between the states when the pulse has reached its maximal amplitude. Different values of $\Delta E_{\mathrm{f}}$ are obtained by adding an offset to $\Delta E_{\mathrm{i}}$, while keeping the length of pulse (thus $\alpha_{\mathrm{si}}$ and the different $\alpha_{j}$ of the "double hat") constant. Since we are interested in finite-time effects, we impose $\Delta E_{\mathrm{f}}<0$ for trapezoid pulses, which reflects that the system is not detuned through the anti-crossing. This condition is relaxed for "double hat" pulses, for which we impose $\Delta E_{\mathrm{f}}<0.5 \mu \mathrm{eV}$. This is equivalent, with our choice of parameters, to letting the system be driven up to the anti-crossing with the slow component of the "double hat". This condition can be written as $\Delta E_{\mathrm{sr}}^{\mathrm{dh}}<0$, i.e. the energy difference at the end of the slow rise-time component is smaller than 0 .

Our results show for the trapezoid pulse what is expected from a finite-time LZSM theory ${ }^{43}$. There is a small probability for an adiabatic transition if the system is detuned to close proximity of the anti-crossing. If one compares this result with values of $P_{\mathrm{a}}$ obtained with the "double hat", it seems that there is no enhancement. Moreover, one would have a tendency to associate values of $P_{\mathrm{a}}$ between $0<\Delta E_{\mathrm{f}}<0.5 \mu \mathrm{eV}$ as originating from the second fast-rise portion of the pulse, which drives the system through the anti-crossing. However, for the particular "double hat" we are considering here, we have $U_{\text {fast } 2} \approx \mathbb{1}$. This means that the values of $P_{\mathrm{a}}$ in the range $0<\Delta E_{\mathrm{f}}<0.5 \mu \mathrm{eV}$ are due to the system being brought close to the anti-crossing with the slow portion of the pulse, for which $\eta \gtrsim 1$. This is in contrast with trapezoid pulses, or any single rise-time pulse, for which $\Delta E_{\mathrm{f}}>0$ implies that most of the magnitude of $P_{\mathrm{a}}$ comes from the

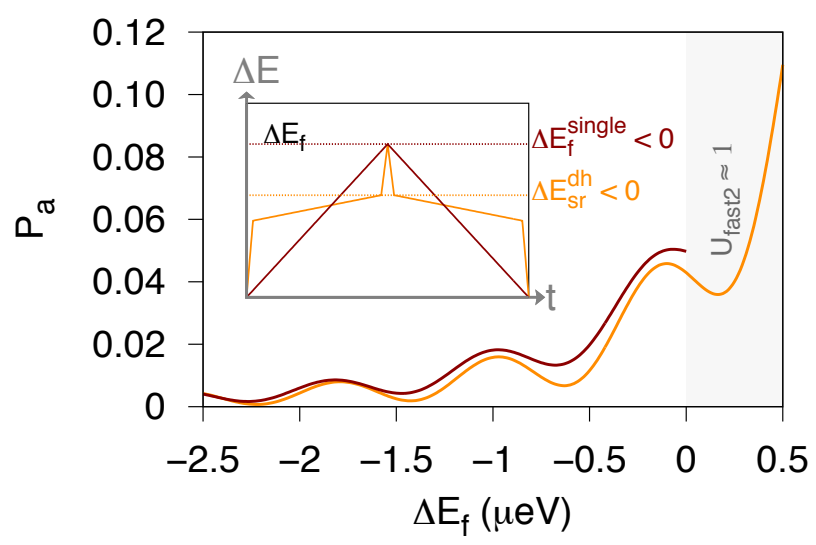

Figure 2. (color online) Comparison of the adiabatic transition probability, $P_{\mathrm{a}}$, for a "double hat" (orange) and a linear pulse (red) with $t_{\mathrm{w}}=0$ as a function of $\Delta E_{\mathrm{f}}$. (Inset) Pulse profiles used to obtain $P_{\mathrm{a}}$. The maximal offset for the single rise-time pulse is chosen such that the system is never driven through the anti-crossing $\left(\Delta E_{\mathrm{f}}^{\text {single }}<0\right)$. The maximal offset for "double hat" pulses is determined by the condition that the system cannot be driven with the slow component of the pulse through the anti-crossing $\left(\Delta E_{\mathrm{sr}}^{\mathrm{dh}}<0\right)$. For "double hat" pulses, the values of $P_{\mathrm{a}}$ between $0<\Delta E_{\mathrm{f}}<0.5 \mu \mathrm{eV}$ originate from the slow portion of the pulse, which brings the system close to the anti-crossing. In this range, the magnitude of $P_{\mathrm{a}}$ is not due to the second fast rise-time portion of the pulse, which drives the system through the anti-crossing.

system being driven through the anti-crossing.

To illustrate our last statement, in Fig. 3 we present a comparison between $P_{\mathrm{a}}$ obtained with a "double hat" pulse as described previously and a "truncated double hat", which is missing the second fast detuning ramp [c.f. inset Fig. 3]. To compare $P_{\mathrm{a}}$ between the two pulses, we choose the $x$-axis to describe $\Delta E_{\mathrm{f}}$ at the end of the slow detuning pulse. We shift the values obtained with a "double hat" along the $x$-axis by an amount $\Delta E_{\text {fast } 2}=$ $0.5 \mu \mathrm{eV}$ to compare between the two different pulses. We clearly see that for both cases $P_{\mathrm{a}}$ is nearly identical.

\section{ADIABATIC CONTROL OF A $\mathrm{S}-\mathrm{T}_{+}$QUBIT}

In the following, we apply the previously developed ideas to a physical implementation of a LZSM driven qubit. We focus on the two-spin $\mathrm{S}-\mathrm{T}_{+}$implementation in a GaAs DQD ${ }^{16117}$, see Fig. 1(b). Although the dynamics of the system under "double hat" pulses has already been studied experimentally ${ }^{18}$, there is still a need to gain a better understanding of the charge-noise-induced spin dephasing. We will show, among other things, that the measurement of finite-time LZSM oscillations can provide a tool to qualitatively access the strength of charge noise. 


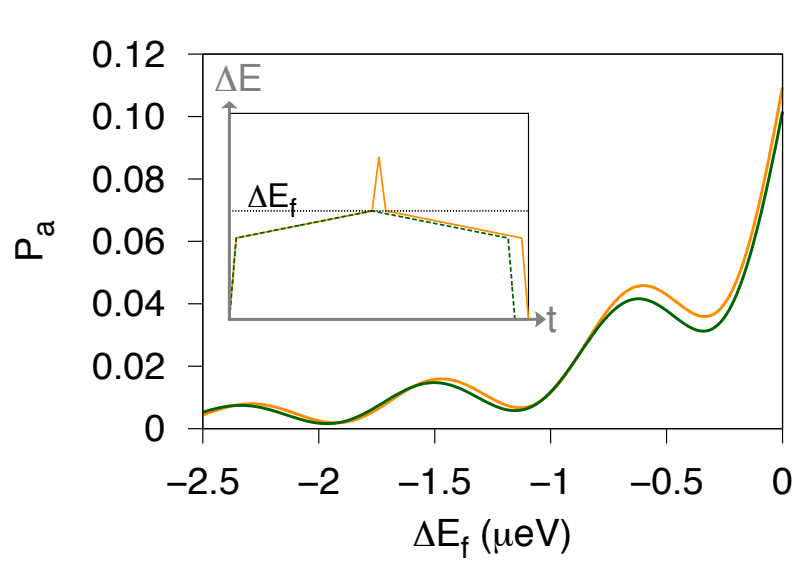

Figure 3. (color online) Comparison of the adiabatic transition probability, $P_{\mathrm{a}}$, for a "double hat" (orange) and a "truncated double hat" (green), which is missing the second fast rise-time component, as a function of $\Delta E_{\mathrm{f}}$ at the end of the slow detuning pulse. (Inset) Pulse profiles used to obtain $P_{\mathrm{a}}$. The results obtained with a "double hat" are shifted by an amount $\Delta E_{\text {fast } 2}$ on the $x$-axis to allow for comparison. We conclude from these results that the state of the system is hardly changed during the second fast detuning pulse.

\section{A. Double Quantum Dot Spin States}

The spin preserving part of the Hamiltonian describing the confinement of electrons in a DQD in the presence of an external magnetic field can be written using a simple two-site hopping model,

$$
\begin{aligned}
H_{0}= & \sum_{\substack{i=1,2 \\
\sigma=\uparrow, \downarrow}}\left(\varepsilon_{i}+\frac{1}{2} g^{*} \mu_{\mathrm{B}} B \sigma\right) c_{i \sigma}^{\dagger} c_{i \sigma}+u \sum_{i} c_{i \uparrow}^{\dagger} c_{i \uparrow} c_{i \downarrow}^{\dagger} c_{i \downarrow} \\
& +\tau \sum_{\sigma}\left(c_{1 \sigma}^{\dagger} c_{2 \sigma}+\text { h.c. }\right) .
\end{aligned}
$$

The index $i=1,2$ labels the dot number and $\sigma=\uparrow, \downarrow=$ \pm 1 the spin of the electron. We denote the energy of a single confined electron by $\varepsilon_{i}$ and the Zeeman energy associated with its spin is given by $g^{*} \mu_{\mathrm{B}} B \sigma / 2$, where $g^{*}$ denotes the effective Landé g-factor, $\mu_{\mathrm{B}}$ the Bohr magneton, $B$ the strength of the external magnetic field. The operators $c_{i \sigma}$ and $c_{i \sigma}^{\dagger}$ describe respectively the annihilation and creation of an electron in $\operatorname{dot} i$ with spin $\sigma$. Two electrons occupying the same QD give rise to an intradot Coulomb energy $u$. The last term of Eq. (8) accounts for electron tunneling between the dots with strength $\tau$. We neglect the inter-dot Coulomb interaction because it only produces a constant shift of the energy levels.

Since most recent experiments on a DQD system are operated in a regime with at most two electrons, we can project Eq. (8) into the subspace spanned by the charge

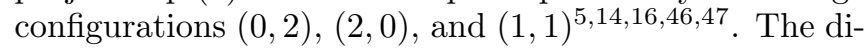
agonalization of the resulting Hamiltonian leads to six low-energy states which are superpositions of the sin-

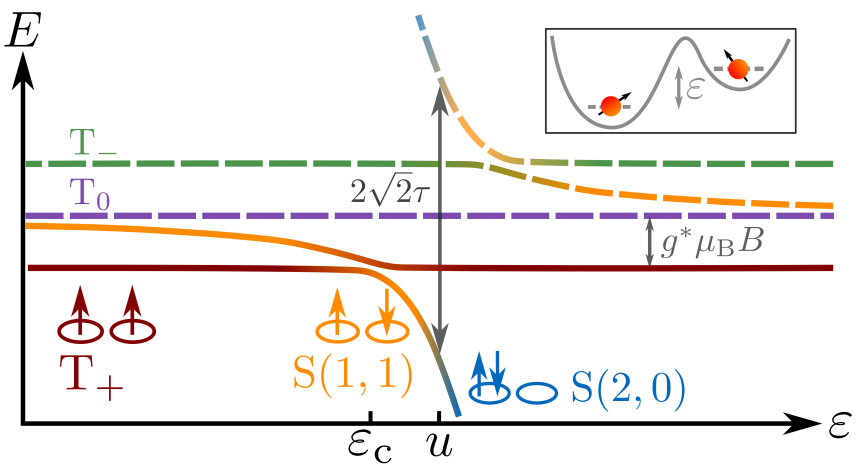

Figure 4. (color online) Energy diagram for the relevant states in the DQD as a function of $\varepsilon$. The spin states for the implementation of the qubit are the hybridized singlet $\mathrm{S}$ and the triplet $\mathrm{T}_{+}$.

glets $\mathrm{S}(0,2), \mathrm{S}(2,0)$, and $\mathrm{S}(1,1)$ as well as the triplets $\mathrm{T}_{0}(1,1), \mathrm{T}_{+}(1,1)$, and $\mathrm{T}_{-}(1,1)$. The triplet states with two particles in the same dot must have electrons occupying higher-energy orbitals due to the Pauli principle. This results in a relatively high separation in energy $(\sim 400 \mu \mathrm{eV})$ from a singlet with both electrons occupying the same dot 48 . Consequently the triplets states with two electrons in the same dot can be safely neglected for the purpose of the current study.

An energy level diagram is shown in Fig. 4, where we plot the energies of the relevant two-electron spin states as a function of detuning $\varepsilon=\varepsilon_{1}-\varepsilon_{2}$. The degeneracy of the singlets $\mathrm{S}(1,1)$ and $\mathrm{S}(2,0)$ at $\varepsilon=u$, as well as between $\mathrm{S}(1,1)$ and $\mathrm{S}(0,2)$ at $\varepsilon=-u$, is lifted due to tunneling, which results in a splitting of the levels by $2 \sqrt{2} \tau$. The degeneracy between the spin singlet $\mathrm{S}$ and spin triplet $\mathrm{T}_{0}$ is lifted due to the exchange interaction $\frac{49}{4}$. This property has allowed the encoding of a spin qubit in these two spin states and its manipulation via the exchange interaction 5 .

Here we concentrate on a particular value of detuning that we have denoted by $\varepsilon_{\mathrm{c}}$ in the energy level diagram. This point corresponds to the crossing of the singlet state with the triplet $\mathrm{T}_{+}$state and is special because there is an anticrossing due to the hyperfine interaction between the electron spins and nuclear spins of the host material. It has been demonstrated experimentally 16 and theoretically 17 that coherent control of the $\mathrm{S}-\mathrm{T}_{+}$qubit can be achieved by detuning the system from an initially prepared $\mathrm{S}(2,0)$ through the hyperfine mediated anti-crossing.

The hyperfine interaction is described by the effective Hamiltonian

$$
H_{\mathrm{HF}}=\boldsymbol{S}_{1} \cdot \boldsymbol{h}_{1}+\boldsymbol{S}_{2} \cdot \boldsymbol{h}_{2}
$$

between the electron spin $\boldsymbol{S}_{i}$ and the effective magnetic fields $\boldsymbol{h}_{i}$ that are generated by the nuclear spins $\boldsymbol{I}_{i}$ in dot $i$. The Overhauser field operators $\boldsymbol{h}_{i}=\sum_{k=1}^{n_{i}} A_{i}^{k} \boldsymbol{I}_{i}^{k}$ describe the nuclear spin bath. Here $n_{i}$ is the number of nuclei in dot $i$ and $A_{i}^{k}=v_{i k} \nu_{0}\left|\psi_{i}\left(\boldsymbol{r}_{k}\right)\right|^{2}$ is the hyperfine coupling constant with the $k$-th nucleus in dot $i$, with 
$\psi_{i}\left(\boldsymbol{r}_{k}\right)$ the electron wave function, $\nu_{0}$ the volume of the unit cell and $v_{i k}$ the hyperfine coupling strength. A more convenient form of the hyperfine interaction is obtained by introducing the spin ladder operators $S_{i}^{ \pm}=S_{i}^{x} \pm \mathrm{i} S_{i}^{y}$ and $h_{i}^{ \pm}=h_{i}^{x} \pm \mathrm{i} h_{i}^{y}$, which yields

$$
H_{\mathrm{HF}}=\frac{1}{2} \sum_{i}\left(2 S_{i}^{z} h_{i}^{z}+S_{i}^{+} h_{i}^{-}+S_{i}^{-} h_{i}^{+}\right) .
$$

The longitudinal part of $H_{\mathrm{HF}}$ is diagonal and its contribution will add to the energy of the triplet state. The transverse part

$$
H_{\mathrm{HF}}^{\perp}=\frac{1}{2} \sum_{i}\left(S_{i}^{+} h_{i}^{-}+S_{i}^{-} h_{i}^{+}\right),
$$

generates the so-called flip-flop process that result in an energy gap at $\varepsilon_{\mathrm{c}}$ and allows for mixing of the $\mathrm{S}$ and $\mathrm{T}_{+}$ spin states $\frac{819 \mid 15}{}$.

\section{B. $\mathrm{S}-\mathrm{T}_{+}$Effective Hamiltonian}

In this section, we derive an effective $2 \times 2$ Hamiltonian that describes the dynamics of the $\mathrm{S}-\mathrm{T}_{+}$spin states near the hyperfine induced anti-crossing. Before doing so, we start by making a few considerations based on LZSM theory to determine which states play a negligible role in the dynamics. Since Eq. (8) describes a series of anti-crossings, it is possible to use the results of Refs. 50 and 51 where a formula for the non-adiabatic transition probability of a multiple crossings LZSM model has been derived. It was shown that for well separated anticrossings 51 , we have $P_{k}=\prod_{j=1}^{k} \exp \left(-2 \pi \lambda_{j}^{2} / \hbar \alpha_{j}\right)$, where $P_{k}$ is the non-adiabatic transition probability after the $k$-th anti-crossing. This formula is a product of $k$ LZSM probabilities, which reflects the independence between the set of anti-crossings.

This model can directly be applied to the DQD system for magnetic fields on the order of a few hundreds of $\mathrm{mT}$, where the charge anti-crossing and both of the hyperfine induced anti-crossings $\left(\mathrm{T}_{+}\right.$and $\left.\mathrm{T}_{-}\right)$are well separated. Here, we demonstrate that if the system is initialized in the singlet $\mathrm{S}(2,0)$, then the detuning pulses allowing for mixing of the lowest energy hybridized singlet state and triplet $\mathrm{T}_{+}$cannot populate the higher energy hybridized singlet state and consequently the triplet $\mathrm{T}_{-}$. Let us consider that the hyperfine coupling strength is on the order of a hundred nano-electron volts, $\lambda_{\mathrm{HF}}=100 \mathrm{neV}$. This value is consistent with experimental findings. In Ref. 16, a strength of $60 \mathrm{neV}$ has been reported. For our choice of $\lambda_{\mathrm{HF}}$, we find $P_{\mathrm{LZSM}}=0.5$ for $\alpha \simeq 138 \mathrm{eVs}^{-1}$. This would correspond to an equally weighted superposition of the qubit states. However, this is only true if there is no population transfer to the higher hybridized singlet level as predicted by the equation for $P_{k}$. By evaluating the population transfer between $\mathrm{S}(2,0)$ and $\mathrm{S}(1,1)$ with the LZSM formula for $\alpha=138 \mathrm{eVs}^{-1}$ and $\lambda_{\text {charge }}=\sqrt{2} \tau \simeq 7.1 \mu \mathrm{eV}$, we find $P_{\mathrm{LZSM}} \simeq 0$, within the numerical precision of our calculation, which indicates that there is no population transfer.

From the previous considerations, we have shown that it is safe to neglect the higher energy hybridized singlet state as well as the triplet $\mathrm{T}_{-}$, but the importance of $\mathrm{T}_{0}$ remains to be determined. Here, we rely on recent experimental results $\frac{47}{4}$ which demonstrate that only a certain type of detuning pulses lead to mixing between $T_{+}, T_{0}$, and the ground-state singlet. Moreover, as the results of Ref. 47 indicate, it is possible to identify in interference patterns the presence of $\mathrm{T}_{0}$ in the dynamics ${ }^{52}$.

Finally, we conclude that it is possible to restrict the Hilbert space to two states, $\mathrm{T}_{+}(1,1)$ and the lowest energy hybridized singlet $\mathrm{S}$. In order to derive an analytical expression for the latter, we start by considering the projection of Eq. (8) onto the states $\mathrm{T}_{+}(1,1), \mathrm{S}(1,1)$, and $\mathrm{S}(2,0)$. We find

$$
H_{0}(\varepsilon)=\left(\begin{array}{ccc}
g^{*} \mu_{\mathrm{B}} B & 0 & 0 \\
0 & 0 & \sqrt{2} \tau \\
0 & \sqrt{2} \tau & u-\varepsilon
\end{array}\right) .
$$

The diagonalization of Eq. 12 yields two hybridized singlets and the triplet state $\mathrm{T}_{+}$

$$
\begin{aligned}
& |\mathrm{S}\rangle=c(\varepsilon)|\mathrm{S}(1,1)\rangle+\sqrt{1-c(\varepsilon)^{2}}|\mathrm{~S}(2,0)\rangle, \\
& \left|\mathrm{S}^{\prime}\right\rangle=c^{\prime}(\varepsilon)|\mathrm{S}(1,1)\rangle+\sqrt{1-c^{\prime}(\varepsilon)^{2}}|\mathrm{~S}(2,0)\rangle, \\
& |\mathrm{T}\rangle=\left|\mathrm{T}_{+}(1,1)\right\rangle
\end{aligned}
$$

with respective energies

$$
\begin{aligned}
& E_{\mathrm{S}}(\varepsilon)=\frac{1}{2}\left(u-\varepsilon-\sqrt{8 \tau^{2}+(u-\varepsilon)^{2}}\right), \\
& E_{\mathrm{S}^{\prime}}(\varepsilon)=\frac{1}{2}\left(u-\varepsilon+\sqrt{8 \tau^{2}+(u-\varepsilon)^{2}}\right), \\
& E_{\mathrm{T}}=g^{*} \mu_{\mathrm{B}} B .
\end{aligned}
$$

The charge admixture coefficients $c(\varepsilon)$ and $c^{\prime}(\varepsilon)$ are

$$
\begin{aligned}
c(\varepsilon) & =\frac{\varepsilon-u-\sqrt{8 \tau^{2}+(u-\varepsilon)^{2}}}{\sqrt{8 \tau^{2}+\left(-\varepsilon+u+\sqrt{8 \tau^{2}+(\varepsilon-u)^{2}}\right)^{2}}}, \\
c^{\prime}(\varepsilon) & =\frac{\varepsilon-u+\sqrt{8 \tau^{2}+(u-\varepsilon)^{2}}}{\sqrt{8 \tau^{2}+\left(\varepsilon-u+\sqrt{8 \tau^{2}+(\varepsilon-u)^{2}}\right)^{2}}} .
\end{aligned}
$$

We can now make the following basis change

$$
T(\varepsilon)=\left(\begin{array}{ccc}
1 & 0 & 0 \\
0 & c(\varepsilon) & c^{\prime}(\varepsilon) \\
0 & \sqrt{1-c^{2}(\varepsilon)} & \sqrt{1-c^{\prime 2}(\varepsilon)}
\end{array}\right),
$$

such that

$$
\begin{aligned}
H_{0}^{\text {diag }}(\varepsilon) & =T(\varepsilon)^{\dagger} H_{0}(\varepsilon) T(\varepsilon) \\
& =\left(\begin{array}{ccc}
g^{*} \mu_{\mathrm{B}} B & 0 & 0 \\
0 & E_{\mathrm{S}} & 0 \\
0 & 0 & E_{\mathrm{S}^{\prime}}
\end{array}\right) .
\end{aligned}
$$




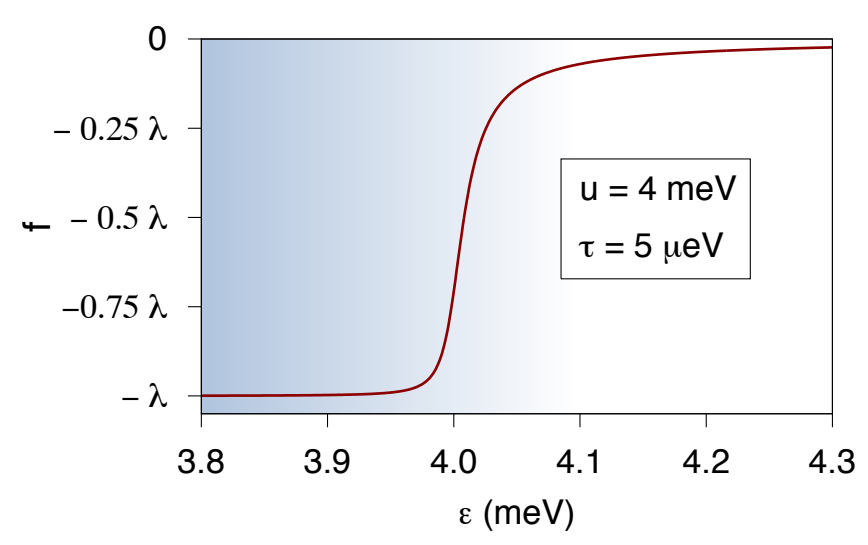

Figure 5. (color online) The effective hyperfine mediated coupling between $|\mathrm{S}\rangle$ and $|\mathrm{T}\rangle$ is a function of detuning. This is a consequence of the state $|S\rangle$ being a superposition of different charge states.

We add now to the Hamiltonian defined in Eq. (12) the hyperfine interaction defined in Eq. (9). Since we are not interested in describing the nuclear spins dynamics, but its effect on the two-spin states $\mathrm{S}$ and $\mathrm{T}_{+}$, we can model the action of the Overhauser field operators by introducing a classical stochastic variable which accounts for fluctuations in the nuclear spin ensemble $e^{8 / 9[15}$. By setting

$$
\boldsymbol{h}_{i}=g^{*} \mu_{\mathrm{B}} \mathbf{B}_{\mathrm{n}, i},
$$

we can interpret $\mathbf{B}_{\mathrm{n}, i}$ as the effective random magnetic field acting on $\boldsymbol{S}_{i}$. Under normal experimental conditions we have $k_{\mathrm{B}} T \gg g_{\mathrm{n}} \mu_{\mathrm{n}} B$, where $g_{\mathrm{n}}$ and $\mu_{\mathrm{n}}$ are the nuclear $g$-factor and magneton. The nuclear spins can, in this limit, be assumed to be completely unpolarized, resulting in a Gaussian distribution of nuclear fields $\$ 8|9| 15$

$$
p\left(B_{\mathrm{n}, i}\right)=\frac{1}{\sqrt{2 \pi} \sigma} \mathrm{e}^{-\frac{B_{\mathrm{n}, i}^{2}}{2 \delta^{2}}},
$$

with $\delta=A / g^{*} \mu_{\mathrm{B}} \sqrt{n}$, the hyperfine coupling constant $A \approx 90 \mu \mathrm{eV}$, and the approximate number of nuclei overlapping with the electronic wave function $n \approx 10^{5}-10^{6}$. By defining $B_{\mathrm{n}, i}^{ \pm}=B_{\mathrm{n}, i}^{x} \pm \mathrm{i} B_{\mathrm{n}, i}^{y}$, the hyperfine Hamiltonian can be written by analogy with Eq. 10 as

$$
H_{\mathrm{HF}}=\frac{1}{2} \sum_{i}\left(2 S_{i}^{z} B_{\mathrm{n}, i}^{z}+S_{i}^{+} B_{\mathrm{n}, i}^{-}+S_{i}^{-} B_{\mathrm{n}, i}^{+}\right)
$$

The longitudinal part of $H_{\mathrm{HF}}$ can be included in the energy of the triplet state $g^{*} \mu_{\mathrm{B}} B \rightarrow g^{*} \mu_{\mathrm{B}}\left(B+B_{\mathrm{n}, 1}^{z}+\right.$ $B_{\mathrm{n}, 2}^{z}$ ), while the transverse part

$$
H_{\mathrm{HF}}^{\perp}=\frac{1}{2} \sum_{i}\left(S_{i}^{+} B_{\mathrm{n}, i}^{-}+S_{i}^{-} B_{\mathrm{n}, i}^{+}\right) .
$$

mixes the spin states $|S\rangle$ and $|\mathrm{T}\rangle$. We therefore find that the full Hamiltonian, in the $\left\{\mathrm{T}_{+}(1,1), \mathrm{S}(1,1), \mathrm{S}(2,0)\right\}$ basis, describing the dynamics of the singlet $\mathrm{S}$ and triplet
$\mathrm{T}_{+}$near the hyperfine anti-crossing is given by

$$
H_{\mathrm{S}-\mathrm{T}_{+}}(\varepsilon)=\left(\begin{array}{ccc}
g^{*} \mu_{\mathrm{B}} \tilde{B} & \lambda & 0 \\
\lambda & 0 & \sqrt{2} \tau \\
0 & \sqrt{2} \tau & (u-\varepsilon)
\end{array}\right),
$$

where $\tilde{B}=B+B_{\mathrm{n}, 1}^{z}+B_{\mathrm{n}, 2}^{z}$ and

$$
\lambda=\left\langle\mathrm{S}(1,1)\left|H_{\mathrm{HF}}^{\perp}\right| \mathrm{T}_{+}(1,1)\right\rangle=g^{*} \mu_{\mathrm{B}}\left(B_{\mathrm{n}, 2}^{-}-B_{\mathrm{n}, 1}^{-}\right) / 2 \sqrt{2} .
$$

In the basis that diagonalizes Eq. 12, the Hamiltonian defined in Eq. 27) reads

$$
\tilde{H}_{\mathrm{S}-\mathrm{T}_{+}}(\varepsilon)=\left(\begin{array}{ccc}
g^{*} \mu_{\mathrm{B}} \tilde{B} & c(\varepsilon) \lambda & c^{\prime}(\varepsilon) \lambda \\
c(\varepsilon) \lambda & E_{\mathrm{S}}(\varepsilon) & 0 \\
c^{\prime}(\varepsilon) \lambda & 0 & E_{\mathrm{S}^{\prime}}(\varepsilon)
\end{array}\right) .
$$

The projection of Eq. 29 onto the Hilbert space spanned by $\{|\mathrm{S}\rangle,|\mathrm{T}\rangle\}$ leads to the effective $2 \times 2$ Hamiltonian describing the dynamics at the singlet-triplet $\mathrm{T}_{+}$ anti-crossing. Taking into account that the detuning is time-dependent, $\varepsilon=\varepsilon(t)$, we finally obtain

$H(t)=E_{\mathrm{S}}(\varepsilon(t))|\mathrm{S}\rangle\left\langle\mathrm{S}\left|+\tilde{E}_{\mathrm{T}}\right| \mathrm{T}\right\rangle\langle\mathrm{T}|+f(\varepsilon(t))(|\mathrm{S}\rangle\langle\mathrm{T}|+$ h.c. $)$,

where $E_{\mathrm{S}}$ is defined in Eq. 16 and $\tilde{E}_{\mathrm{T}}=g^{*} \mu_{\mathrm{B}} \tilde{B}$. This effective Hamiltonian differs from previous derivations ${ }^{17 / 53}$ in the coupling $f(t)=c(\varepsilon(t)) \lambda$, which is timedependent. Here, $c(\varepsilon(t))$ and $\lambda$ are given in Eqs. 19p and $(28)$. As it can be seen from the functional form of $f(t)$, the effective coupling strength between the spin states depends on the charge state (Fig. 5). This result is rather natural since the matrix element between $\mathrm{S}$ and $\mathrm{T}_{+}$goes to zero when the detuning is such that $\mathrm{S}=\mathrm{S}(2,0)$ and $\mathrm{T}_{+}=\mathrm{T}_{+}(1,1)$, i.e., $\left\langle\mathrm{S}\left|H_{\mathrm{HF}}^{\perp}\right| \mathrm{T}_{+}\right\rangle \rightarrow 0$ for $\varepsilon \gg u$. It also implies that the physics described by Eq. (30) goes beyond standard LZSM theory with a constant coupling. However, since both Hamiltonians describe adiabatic passage through an anti-crossing, we can assume that the dynamics are qualitatively similar. We can therefore expect that our previous discussion about enhancement of adiabaticity based on LZSM physics remains valid. Furthermore, due to the peculiar form of $f(t)$ (c.f. Fig. 5), we can assume that it is possible to observe finite-time interferometry phenomena in close vicinity of the anti-crossing.

\section{Master Equation}

In order to compare our theory with experimental measurements, it is not sufficient to solve the Hamiltonian dynamics provided by Eq. (30), because some important phenomena that can influence the outcome of the experiment are not taken into account. Among these are spin relaxation due to phonon-assisted hyperfine interaction $\sqrt{54}$ and charge fluctuations that lead to dephasing of the qubit states 55 . These phenomena can be taken into 
account in a quantum master equation formalism for the density matrix. In the Lindblad formalism $56 \mid 57$, the time evolution can be expressed as

$$
\dot{\rho}=-\frac{\mathrm{i}}{\hbar}[H, \rho]+\frac{1}{2} \sum_{j=1}^{N^{2}-1}\left(\left[L_{j} \rho, L_{j}^{\dagger}\right]+\left[L_{j}, \rho L_{j}^{\dagger}\right]\right) .
$$

The operators $L_{j}$ are called Lindblad operators 56157 and they describe the dissipative effect of the environment on the system in the Born-Markov approximation, which consists of two assumptions that lead to Eq. (31). The Born approximation supposes a weak coupling between the system and the bath, while the Markov approximation consists in neglecting any type of memory effects of the bath during the system evolution. To fully describe the effect of the environment, one needs $N^{2}-1$ operators where $N$ is the dimension of the system's Hilbert space 56157 . For the case of a two-level system, the Lindblad operators are $L_{1}=\sqrt{\Gamma_{-}} \sigma_{-}, L_{2}=\sqrt{\Gamma_{+}} \sigma_{+}$, and $L_{3}=\sqrt{\Gamma_{\varphi}} \sigma_{z}$, where $\sigma_{-}$and $\sigma_{+}$are spin ladder operators and $\sigma_{z}$ is the $z$ Pauli matrix. They respectively describe relaxation from the excited state to the ground state with rate $\Gamma_{-}$, relaxation from the ground state to the excited state with rate $\Gamma_{+}$, and pure dephasing with rate $\Gamma_{\varphi}$.

By substituting Eq. (30) into Eq. (31) and using the expression of the Lindblad operators, the first order differential equation for the $\mathrm{S}-\mathrm{T}_{+}$density matrix can be written as

$$
\left(\begin{array}{l}
\dot{\rho}_{11} \\
\dot{\rho}_{12} \\
\dot{\rho}_{21} \\
\dot{\rho}_{22}
\end{array}\right)=\left(\begin{array}{cc}
-\Gamma_{+} & \frac{\mathrm{i}}{\hbar} f(t) \\
\frac{\mathrm{i}}{\hbar} f(t) & -\frac{\mathrm{i}}{\hbar}\left(E_{\mathrm{S}}(t)-\tilde{E}_{\mathrm{T}}\right)-\frac{1}{2}\left(\Gamma_{+}+\Gamma_{-}+4 \Gamma_{\varphi}\right) \\
-\frac{\mathrm{i}}{\hbar} f(t) & 0 \\
\Gamma_{+} & -\frac{\mathrm{i}}{\hbar} f(t)
\end{array}\right.
$$

$$
\left.\begin{array}{cc}
-\frac{\mathrm{i}}{\hbar} f(t) & \Gamma_{-} \\
0 & -\frac{\mathrm{i}}{\hbar} f(t) \\
\frac{\mathrm{i}}{\hbar}\left(E_{\mathrm{S}}(t)-\tilde{E}_{\mathrm{T}}\right)-\frac{1}{2}\left(\Gamma_{+}+\Gamma_{-}+4 \Gamma_{\varphi}\right) & \frac{\mathrm{i}}{\hbar} f(t) \\
\frac{\mathrm{i}}{\hbar} f(t) & -\Gamma_{-}
\end{array}\right)\left(\begin{array}{c}
\rho_{11} \\
\rho_{12} \\
\rho_{21} \\
\rho_{22}
\end{array}\right) .
$$

This system of four coupled ordinary differential complex equations can be reduced to a system of three coupled ordinary differential equations (Bloch equations) by introducing new real variables defined by

$$
\begin{aligned}
& x=\rho_{12}+\rho_{21}, \\
& y=\mathrm{i}\left(\rho_{12}-\rho_{21}\right), \\
& z=\rho_{11}-\rho_{22} .
\end{aligned}
$$

This set of variables is completed by the conservation of probability condition,

$$
\rho_{11}+\rho_{22}=1 .
$$

Substituting Eqs. (33) and (34) into Eq. (32), one finds the system of ordinary differential equations for the new variables,

$$
\begin{aligned}
& \dot{x}=-\frac{E_{\mathrm{S}}-\tilde{E}_{\mathrm{T}}}{\hbar} y-\frac{1}{2}\left(\Gamma_{+}+\Gamma_{-}\right) x-2 \Gamma_{\varphi} x, \\
& \dot{y}=\frac{E_{\mathrm{S}}-\tilde{E}_{\mathrm{T}}}{\hbar} x-2 \frac{f}{\hbar} z-\frac{1}{2}\left(\Gamma_{+}+\Gamma_{-}\right) y-2 \Gamma_{\varphi} y, \\
& \dot{z}=2 \frac{f}{\hbar} y-\left(\Gamma_{+}+\Gamma_{-}\right) z+\Gamma_{-}-\Gamma_{+} .
\end{aligned}
$$

Here, we assume that relaxation occurs through phonon-assisted hyperfine interaction. Since we are dealing with small energy transfers, we consider only piezo phonons 58 . The Hamiltonian describing the coupling between the logical qubit states (i.e. the instantaneous energy eigenstates of Eq. (30) and the phonons is given by

$$
\begin{aligned}
& H_{\mathrm{qp}}=\sigma_{z} \otimes U_{\mathrm{ph}}, \text { with } \\
& U_{\mathrm{ph}}(\boldsymbol{r}, t)=\sum_{\nu, \boldsymbol{q}} \sqrt{\frac{\hbar}{2 \rho \omega_{\nu, \boldsymbol{q}}}} A_{\nu, \boldsymbol{q}}\left[\mathrm{e}^{\mathrm{i}\left(\boldsymbol{q} \cdot \boldsymbol{r}-\omega_{\nu, \boldsymbol{q}} t\right)} b_{\nu, \boldsymbol{q}}^{\dagger}+h . c .\right],
\end{aligned}
$$

and $\sigma_{z}$ is the pseudospin $z$-Pauli matrix for $|\mathrm{S}\rangle$ and $|\mathrm{T}\rangle$. Here, $b_{\nu, \boldsymbol{q}}^{\dagger}\left(b_{\nu, \boldsymbol{q}}\right)$ creates (annihilates) a phonon with polarization $\nu$ and wave vector $\boldsymbol{q} . A_{\nu, \boldsymbol{q}}$ is the effective piezoelectric modulus, which depends only on the direction of $q$.

The rates $\Gamma_{+}$and $\Gamma_{-}$can be derived using Redfield theory ${ }^{59160}$. We have

$$
\Gamma_{ \pm}=4\left|\left\langle 0\left|\sigma_{z}\right| 1\right\rangle\right|^{2} J_{ \pm}(\omega)
$$

where $|0\rangle$ and $|1\rangle$ are the qubit states (i.e. in our case the eigenstates of Eq. (30)), $\omega$ the angular frequency defined by the difference in energy of the qubit states, and the spectral densities $J_{ \pm}(\omega)$ are defined by

$$
J_{ \pm}(\omega)=\int_{0}^{\infty} \mathrm{d} t \mathrm{e}^{\mp \mathrm{i} \omega t}\left\langle U_{\mathrm{ph}}(\boldsymbol{r}, 0) U_{\mathrm{ph}}(\boldsymbol{r}, t)\right\rangle .
$$

Here, $\langle\ldots\rangle=\operatorname{Tr}\left[\ldots \rho_{\mathrm{ph}}\right]$ with $\rho_{\mathrm{ph}}$ the density matrix of the phonon bath at thermal equilibrium.

The evaluation of Eq. (40) leads to

$$
J_{+}(\omega)=\sum_{\nu} \frac{3\left\langle A^{2}\right\rangle}{4 \pi^{2} \rho c_{\nu}^{3} \hbar} \omega n(\omega)
$$

and

$$
J_{-}(\omega)=\sum_{\nu} \frac{3\left\langle A^{2}\right\rangle}{4 \pi^{2} \rho c_{\nu}^{3} \hbar} \omega(1+n(\omega))
$$




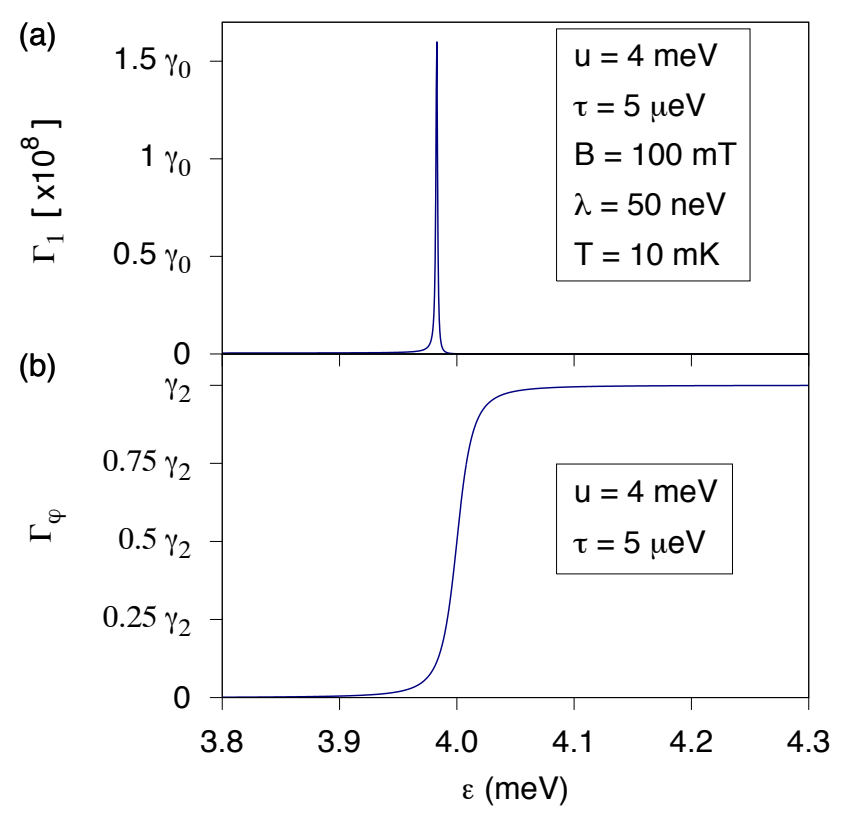

Figure 6. (color online) (a) Relaxation rate $\Gamma_{1}(\varepsilon)$ obtained for $B=100 \mathrm{mT}$. Here, we consider hyperfine mediated flip-flops of the electron spin accompanied by emission or absorption of phonons. The relaxation rate is maximal for $\varepsilon=\varepsilon_{\mathrm{c}}$ where $\left|E_{\mathrm{S}}^{d}(\varepsilon)-E_{\mathrm{T}}^{d}\right| \ll k_{\mathrm{B}} T$. This results in a strong mixing of the spin states due to thermal fluctuations. (b) Charge noise induced dephasing rate $\Gamma_{2}(\varepsilon)$. The dephasing rate is assumed to have a functional dependence proportional to $1-c(\varepsilon)^{2}$ to ensure that dephasing related to charge noise is weaker when the system is in a $(1,1)$ charge configuration.

with $c_{\nu}$ the sound velocity and $n(\omega)$ the Bose-Einstein occupation number, $n(\omega)=(\exp (\beta \hbar \omega)-1)^{-1}$, where $\beta=1 / k_{\mathrm{B}} T . k_{\mathrm{B}}$ is the Boltzman's constant and $T$ is the phonon bath temperature, and $\left\langle A^{2}\right\rangle$ denotes an average piezoelectric modulus. In the following, we denote the result of the sum over $\nu$ by $\gamma_{0}$.

Since $\Gamma_{+}$and $\Gamma_{-}$can be related to each other by considering the limiting case of thermal equilibrium, Eqs. (37) can be simplified to include only two inde- pendent rates. If the system reaches thermal equilibrium then the detailed balance equation $\rho_{11}^{\mathrm{th}} \Gamma_{+}=\rho_{22}^{\mathrm{th}} \Gamma_{-}$ holds. Moreover, the populations are given by the canonical ensemble, $\rho_{i i}^{\text {th }}=\exp \left(-\beta E_{i}\right) / Z$, with $Z$ the partition function. This yields

$$
\frac{\Gamma_{+}}{\Gamma_{-}}=\frac{\rho_{22}^{\mathrm{th}}}{\rho_{11}^{\mathrm{th}}}=\mathrm{e}^{-\beta \hbar \omega}
$$

where we used $\hbar \omega=\sqrt{\left(E_{\mathrm{S}}(\varepsilon)-\tilde{E}_{\mathrm{T}}\right)^{2}+4 f^{2}(\varepsilon)}$, which is the energy difference between the eigenstates of Eq. 30 .

Combining the results of Eqs. (39), 441), 442), and (43), we find that

$$
\Gamma_{1}(\varepsilon)=\Gamma_{+}+\Gamma_{-}=\gamma_{0} \frac{f^{2}(\varepsilon)}{\hbar^{2} \omega} \operatorname{coth}\left(\frac{\hbar \omega}{2 k_{\mathrm{B}} T}\right) .
$$

This function is plotted against $\varepsilon$ in Fig. 6)(a), and has a peak at $\varepsilon=\varepsilon_{\mathrm{c}}$ where $\hbar \omega \ll k_{\mathrm{B}} T$ resulting in a strong mixing of the states due to thermal fluctuations.

Pure dephasing can originate from orbital effects. In our current description of the problem, we have neglected that the wave functions of the singlet $|\mathrm{S}(2,0)\rangle$ and triplet $\left|\mathrm{T}_{+}(1,1)\right\rangle$ couple differently to the background charge environment due to their different charge configurations. Thus, a superposition state of the form $|\psi\rangle=\alpha|\mathrm{S}(2,0)\rangle+\beta\left|\mathrm{T}_{+}(1,1)\right\rangle$ is sensitive to background charge fluctuations (charge noise), which leads to dephas-

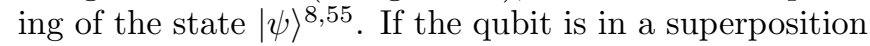
with same charge state, $|\varphi\rangle=\alpha|\mathrm{S}(1,1)\rangle+\beta\left|\mathrm{T}_{+}(1,1)\right\rangle$, then the effects of charge noise are assumed to become weaker. Therefore, we choose to write the charge induced dephasing as

$$
\Gamma_{\varphi}(\varepsilon)=\gamma_{2}\left(1-|c(\varepsilon)|^{2}\right),
$$

where $\gamma_{2}$ is the charge noise rate. In Fig. 6, we plot the rates $\Gamma_{1}(\varepsilon)$ and $\Gamma_{\varphi}(\varepsilon)$.

Finally, the Bloch equations, written in a matrix form, and describing the dynamics around the $\mathrm{S}-\mathrm{T}_{+}$anticrossing are

$$
\hbar\left(\begin{array}{c}
\dot{x} \\
\dot{y} \\
\dot{z}
\end{array}\right)=\left(\begin{array}{ccc}
-\frac{1}{2} \hbar\left(\Gamma_{1}(\varepsilon(t))+4 \Gamma_{\varphi}(\varepsilon(t))\right) & -E_{\mathrm{S}}(\varepsilon(t))+\tilde{E}_{\mathrm{T}} & 0 \\
E_{\mathrm{S}}(\varepsilon(t))-\tilde{E}_{\mathrm{T}} & -\frac{1}{2} \hbar\left(\Gamma_{1}(\varepsilon(t))+2 \Gamma_{\varphi}(\varepsilon(t))\right) & -2 \lambda c(\varepsilon(t)) \\
0 & 2 \lambda c(\varepsilon(t)) & -\hbar \Gamma_{1}(\varepsilon(t))
\end{array}\right)\left(\begin{array}{c}
x \\
y \\
z
\end{array}\right) \pm \hbar\left(\begin{array}{c}
0 \\
0 \\
\gamma_{1}
\end{array}\right) .
$$

The different signs in front of the inhomogeneous term come from the fact that the states $|S\rangle$ and $|T\rangle$ exchange their roles as ground and excited state of the system at $\varepsilon=\varepsilon_{\mathrm{c}}$. The spontaneous relaxation rate $\gamma_{1}$ is given by

$$
\gamma_{1}=\gamma_{0} \frac{f^{2}(\varepsilon)}{\hbar^{2} \omega}
$$

\section{RESULTS}

In the following we first present a comparison between experimental and theory results obtained with experimental pulse profiles, which were measured at the output port of the waveform generator, and experimentally determined $E_{\mathrm{S}}(\varepsilon)$ and $c(\varepsilon)$. We then show further theory results for which we study the effect of charge dynam- 
ics and phonon-mediated hyperfine relaxation. For this purpose, we have solved Eq. (46) for different $\gamma_{0}$ 's and $\gamma_{2}$ 's.

The singlet return probability is obtained by solving Eq. (46) for different realizations of $\lambda$. The average value of $P_{\mathrm{S}}$ is then calculated according to

$$
P_{\mathrm{S}}=\frac{1}{N} \sum_{i=1}^{N} \frac{1}{2}\left(1+z^{(i)}\right),
$$

where $z^{(i)}$ is the $i$ th solution of $z(t)$ in Eq. 46 .

\section{A. Experimental observation of finite-time effects}

We consider a "double hat" detuning pulse whose profile is shown in the inset of Fig. 7(a). The leading edge has a rise-time of $0.1 \mathrm{~ns}$ and an amplitude $A_{1 \mathrm{f}}$, which is followed by a slow ramp with rise-time $t_{\text {slow }}$ and amplitude $A_{\mathrm{s}}=-0.065 \mathrm{meV}$. A $0.1 \mathrm{~ns}$ rise-time pulse shifts the detuning to its maximal value of $-0.39 \mathrm{meV}$, where the detuning is held constant for a time interval $t_{\mathrm{w}}$. The trailing edge of the pulse is simply the reverse of the leading edge. The conversion between gate voltage and energy is performed using the measured lever-arm $\sim 0.13 \mathrm{meV} / \mathrm{mV} 2961$. In Fig. 7, we compare experiment and theory for $t_{\text {slow }}=4 \mathrm{~ns}, A_{1 \mathrm{f}}=-0.26 \mathrm{meV}$.

$P_{\mathrm{S}}$ is plotted as a function of $t_{\mathrm{w}}$ and $\varepsilon_{\mathrm{s}}$ for $B=55 \mathrm{mT}$ in Fig. 7(a) and (b). A "spin-funnel" that is obtained using the spectroscopy method developed in Ref. 5 with a waiting time $t_{\mathrm{w}}=20 \mathrm{~ns}$ is shown in Fig. 77(c) along with the corresponding theory plot in Fig. $7(\mathrm{~d})$. Since the experimental cycles have a short period of $5 \mu \mathrm{s}$, there is a build up of nuclear polarization that generates a gradient field 14. To take this into account in our model, we add a mean to the nuclear field distributions Eq. 24) . It is sufficient to consider only a mean $\xi_{1}^{x}$ for $B_{n, 1}^{x}$ because only the magnitude of the gradient field plays a role in the dynamics. This can be easily understood by considering a rotation about the $z$-axis of the coordinate system that brings the $x$-axis to coincide with the direction of $\boldsymbol{B}_{\mathrm{n}, 2}^{\perp}-$ $\boldsymbol{B}_{\mathrm{n}, 1}^{\perp}$. Parameters used in the theory panels are $\delta=1 \mathrm{mT}$, $\xi_{1}^{x}=10 \mathrm{mT}, \gamma_{0}=10^{-2}$ and $\gamma_{2}=10^{8} \mathrm{~s}^{-1}$.

Both the experimental results and numerical simulations show enhanced interference visibility within the region between $\varepsilon_{\mathrm{S}} \sim-0.19 \mathrm{meV}$ and $\sim-0.29 \mathrm{meV}$. This region should correspond to values of $P_{\mathrm{S}}$ determined by the slow rise-time component of the pulse, as demonstrated in Ref. 18. The position of the anti-crossing is located at $\sim-0.14 \mathrm{meV}$ from the data shown in Fig. 7(a) and (c). Moreover, since $A_{1 \mathrm{f}}=-0.26 \mathrm{meV}, A_{\mathrm{s}}=-0.065 \mathrm{meV}$, and the maximal pulse amplitude is $-0.39 \mathrm{meV}$, the high contrast region should be located between $-0.21 \mathrm{meV}$ and $-0.27 \mathrm{meV}$, in good agreement with our results.

As discussed in Sec. IIB, the visibility of the oscillation pattern contained between $\varepsilon_{\mathrm{s}} \sim-0.14 \mathrm{meV}$ and $\sim-0.21 \mathrm{meV}$ cannot result from the second fast risetime portion of the pulse. Although it drives the system

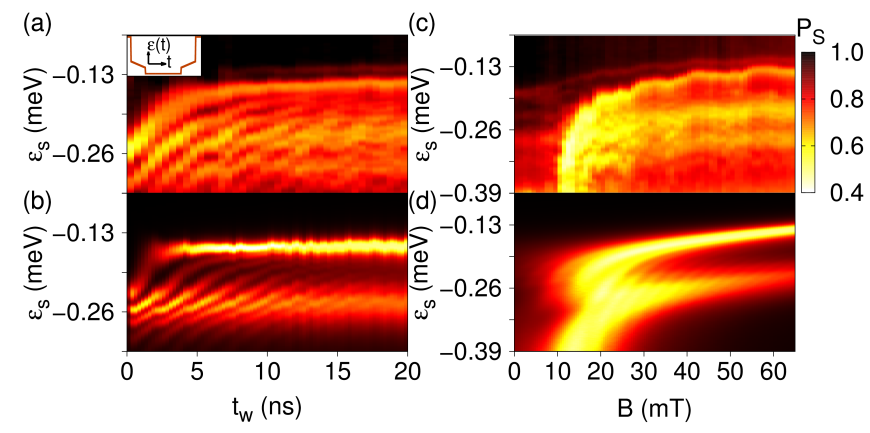

Figure 7. (color online) (a) Measurements of $P_{\mathrm{S}}$ as a function of $t_{\mathrm{w}}$ and $\varepsilon_{\mathrm{s}}$ for $A_{1 \mathrm{f}}=-0.26 \mathrm{meV}, t_{\text {slow }}=4 \mathrm{~ns}$, and $B=$ $55 \mathrm{mT}$. "Double hat" pulses allow the observation of nonadiabatic transitions when the system is driven slowly to close proximity of the anti-crossing. (b) Theoretical predictions obtained using a pulse profile obtained at the output of the waveform generator and parameters from (a). (c) $P_{\mathrm{S}}$ plotted as a function of $B$ and $\varepsilon_{\mathrm{s}}$ for $t_{\mathrm{w}}=20 \mathrm{~ns}$ reveals the spinfunnel. Parameters are $A_{1 \mathrm{f}}=-0.26 \mathrm{meV}$ and $t_{\text {slow }}=4 \mathrm{~ns}$. (d) Theoretical calculations for the same parameters as in (c).

through the anti-crossing, the level velocity is too high to allow for large magnitude non-adiabatic transition probabilities. Thus, as we showed in Figs. 2 and 3 by considering a finite-time LZSM model, we are able to observe a non-adiabatic transition event due to a slow level velocity pulse that brings the system to close vicinity of the anti-crossing, but without driving it through.

To ensure that we are observing finite-time effects, we consider a second "double hat" pulse that has a different $A_{1 \mathrm{f}}$, which changes the relative starting and stopping position of the slow rise-time component of the pulse. As a consequence the relative propagation times $t_{\mathrm{i}}$ and $t_{\mathrm{f}}$, which are defined by setting $t=0$ at the anti-crossing, are also modified.

We consider a second pulse with $A_{1 \mathrm{f}}=-0.29 \mathrm{meV}$, for which the results are presented in Fig. 8. First, we observe that the high-contrast region is shifted towards more positive detunings, as expected. Second, we notice the overall difference between the interference pattern in Figs. 7 and 8 , panels (a) and (b). This dissimilarity can only be explained by different phase accumulation due to distinct $t_{\mathrm{i}}$ and $t_{\mathrm{f}}$. In the usual scattering description of LZSM interferometry, the transition probability only depends on the level velocity at the anti-crossing and on the coupling strength. But here, these two quantities have remained unchanged. These results show the importance of using finite-time models to describe adiabatic passage experiments.

We also anticipate, for LZSM driven qubits, the possibility to manipulate the states by keeping a constant driving and instead change the relative starting and stopping position of the detuning pulse. 


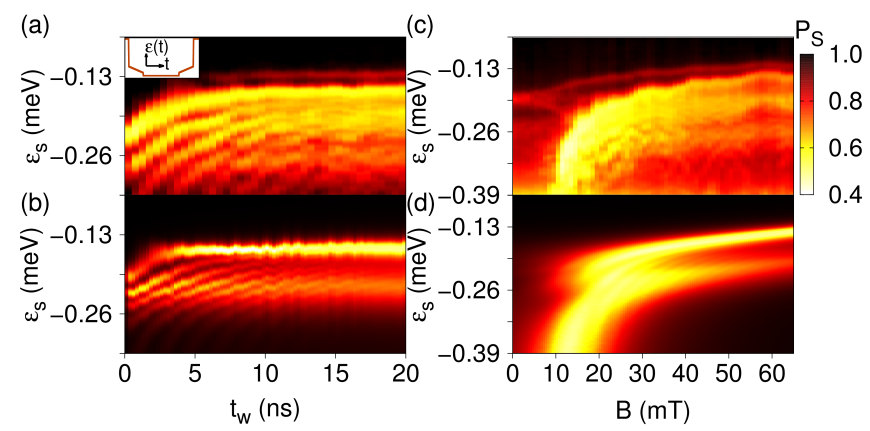

Figure 8. (color online) Experimentally, (a) and (c), and theoretically, (b) and (d), obtained LZSM interference patterns and spin-funnels. Here $A_{1 \mathrm{f}}=-0.29 \mathrm{meV}, t_{\text {slow }}=4 \mathrm{~ns}$, and $B=55 \mathrm{mT}$. The waiting time for the spin-funnel is $t_{\mathrm{w}}=$ $20 \mathrm{~ns}$. The interference patterns differ from results presented in Fig. 7 indicating that the evolution of the system is not only sensitive to the level velocity, but also the time at which the change in level velocity happens.

\section{B. Effects of Phonon-Mediated Hyperfine Relaxation and Charge Induced Dephasing}

In this section, we consider a detuning pulse $\varepsilon(t)$ with an amplitude of $\varepsilon_{\mathrm{s}}-\varepsilon_{\mathrm{i}}=0.2 \mathrm{meV}$. The pulse reaches an amplitude of $0.12 \mathrm{meV}$ in $0.1 \mathrm{~ns}$, it is then slowed down until it reaches an amplitude of $0.1325 \mathrm{meV}$, and finally it is brought to its maximal amplitude in $0.1 \mathrm{~ns}$. The rise time $t_{\text {slow }}$ of the slow part of the pulse can be tuned freely. Here we compute $P_{\mathrm{S}}$ for $t_{\text {slow }}=8$ ns. In Fig. 9(a)-(d), we present the singlet return probability as a function of $\varepsilon_{\mathrm{s}}$ and waiting time $t_{\mathrm{w}}$ for $B=100 \mathrm{mT}, u=4 \mathrm{meV}$, $\tau=5 \mu \mathrm{eV}$, and different values of $\gamma_{0}$ and $\gamma_{2}$. The interference fringes are characterized, for $\varepsilon_{\mathrm{s}}<\varepsilon_{\mathrm{c}}$, by three distinct regions showing an alternate oscillation amplitude for $P_{\mathrm{S}}$. The darker regions coincide with detunings for which the passage through the anti-crossing happened during one of the fast rise-times of $\varepsilon(t)$. Similarly, the bright region between $\varepsilon_{\mathrm{s}} \simeq 3.900-3.915 \mathrm{meV}$ coincides with a passage through the anti-crossing with the slow rise-time portion of the detuning pulse. Experimentally measured interference patterns exhibit identical behavior.

Our results also clearly show coherent evolution of the qubit for $\varepsilon_{\mathrm{s}}>\varepsilon_{\mathrm{c}}$, Fig. 9(e) [blue and purple trace, $\left.\gamma_{2}=10^{8} \mathrm{~s}^{-1}\right]$. This corresponds to the case where the system is not detuned through the anti-crossing. It indicates that it is possible to design complex pulses that can directly influence the competition between LZSM physics and charge noise. However, this is conditional on the dephasing time scale associated with charge noise. We notice that if $\gamma_{2}=10^{9} \mathrm{~s}^{-1}$, then it is impossible to identify any coherent behavior [green and red traces Fig. 7(e)].

Our results indicate that charge noise strongly affects the dynamics while spin relaxation only has a moderate effect. Although this behavior can be identified when comparing Fig. 9(a) with Fig. 9(b) and Fig. 9(c), it is best seen in Fig. 9(f) when comparing traces. An increase in the noise rate $\gamma_{2}$ leads to a substantial decrease of the oscillation visibility (blue and green traces). On the other hand, the visibility is only slightly diminished when relaxation is enhanced (blue and purple traces).

Here, charge noise has a drastic effect on spin dynamics. Since it leads to strong dephasing of the logical qubit states when spin and charge degrees of freedom are correlated, it results in a competition mechanism against LZSM interferometry 62 67. Since the hyperfine mediated anti-crossing is close to the charge anti-crossing for reasonable values of $B$, charge noise also affects the dynamics during the passage through the anti-crossing. As a result, the efficiency of the LZSM mechanism to create a coherent superposition or to produce coherent interferences is hindered, and thus the optimal state populations are not reached. This behavior can be identified in Fig. 9(f) when comparing traces obtained with the same relaxation rate, but different charge noise rates (i.e. blue with green trace and purple with red trace). We clearly identify that the oscillations visibility is smaller for larger values of $\gamma_{2}$.

Energy relaxation processes have a weaker influence on spin dynamics than dephasing due to their dependence on the energy difference between the eigenstates of the system. These become important only when the system is held in close vicinity of the anti-crossing, where relaxation is maximum [c.f. Fig. 6(a)].

These results suggest that inhomogeneous dephasing due to nuclear spin fluctuations is not the only physical process that limits the coherence of a two-spin based qubit, but also that charge noise plays a major role. This is an important result for future devices made out of $\mathrm{Si} / \mathrm{SiGe}$ which are reaching a maturity level comparable to $\mathrm{GaAs}_{58}^{68}$. Silicon based devices are very interesting candidates for spin based quantum computing because the only stable isotope $\left({ }^{29} \mathrm{Si}\right)$ possessing a nuclear spin $(I=1 / 2)$ has relatively low abundance, $\approx 5 \%$. Thus, hyperfine induced decoherence is weaker than in GaAs based nanostructures 68 .

Consequently, we distinguish three time scales that govern the physics of partial spin adiabatic passage in DQDs. There is the rise-time of the detuning pulse, the decoherence time $T_{2}^{*}$, and $T_{\varphi}$ associated with charge noise induced dephasing. Ideally, we would like to have risetimes shorter than $T_{\varphi}$ to preserve any spin superposition state. But this would render adiabatic transitions unlikely and therefore seriously hinder manipulation of the qubit. "Double hat" pulses partially solve the problem, but for an even better result, it would be necessary to increase the coupling between the qubit states. In GaAs double quantum dots, it is possible to prepare a nuclear gradient field to enhance the hyperfine coupling ${ }^{14}$, with the advantage of extending $T_{2}^{*}$. In almost nuclear spin free systems ( $\mathrm{Si}$ or C based DQDs), it is possible to use micro-magnets to artificially induce a coupling between the $\mathrm{S}-\mathrm{T}_{+}$qubit states ${ }^{71}$. 


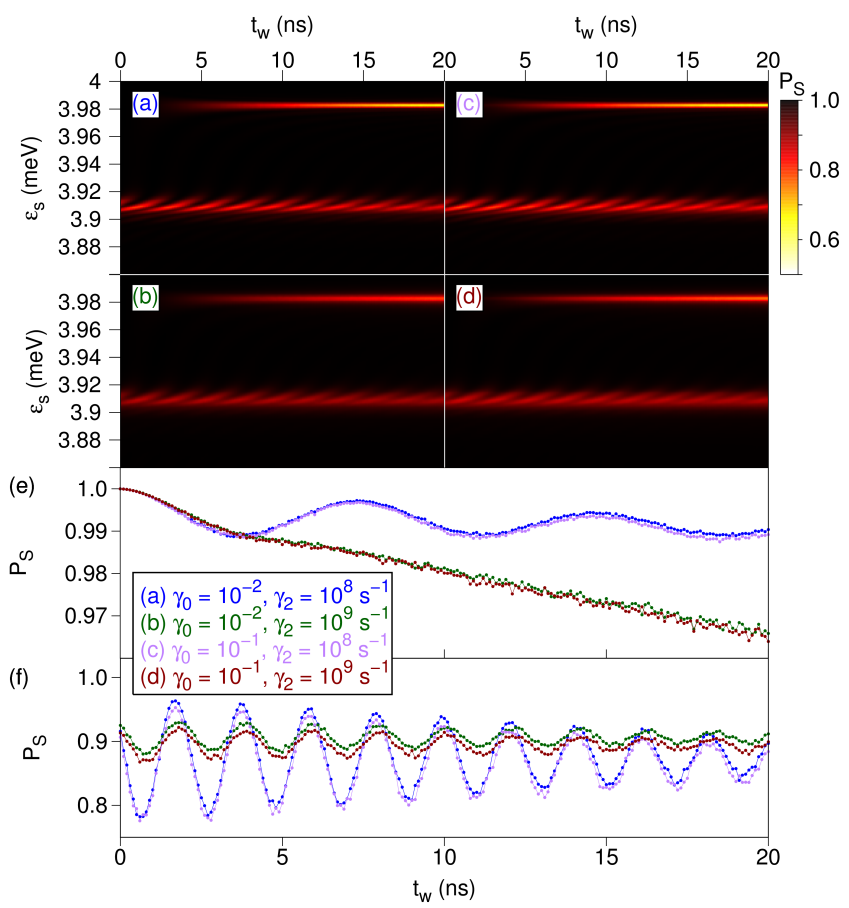

Figure 9. (color online) Singlet return probability $P_{\mathrm{S}}$ as a function of the waiting time $t_{\mathrm{w}}$ and final position $\varepsilon_{\mathrm{s}}$ obtained for a "double hat" detuning pulse. Pulse details are given in the text. Here, we have set $B=100 \mathrm{mT}, u=4 \mathrm{meV}$, and $\tau=5 \mu \mathrm{eV}$ for all plots. The parameters $\gamma_{0}$ and $\gamma_{2}$ are (a) $\gamma_{0}=10^{-2}, \gamma_{2}=10^{8} \mathrm{~s}^{-1}$, (b) $\gamma_{0}=10^{-2}, \gamma_{2}=10^{9} \mathrm{~s}^{-1}$, (c) $\gamma_{0}=10^{-1}, \gamma_{2}=10^{8} \mathrm{~s}^{-1}$, and (d) $\gamma_{0}=10^{-1} \mathrm{~s}^{-1}, \gamma_{2}=$ $10^{9} \mathrm{~s}^{-1}$. Figures (e) and (f) show cuts for case (a) in blue, (b) in dark green, (c) in purple, and (d) in dark red. The cuts are respectively taken before the anti-crossing at $\varepsilon_{\mathrm{s}}=3.987 \mathrm{meV}$ and after at $\varepsilon_{\mathrm{s}}=3.908 \mathrm{meV}$.

\section{CONCLUSIONS}

We have developed a master equation formalism to study the dynamics of a $\mathrm{S}_{-} \mathrm{T}_{+}$qubit in the vicinity of the hyperfine mediated anti-crossing. In comparison with previous theories that only included decoherence due to the hyperfine interaction with nuclear spins, we also include phonon-mediated hyperfine spin relaxation and spin dephasing due to charge noise. We have also derived a new effective two-level spin-charge Hamiltonian. In addition to previous theories, we take into account the charge degree of freedom. This property originates from the lowest energy singlet state $|S\rangle$ being a superposition of different charge configurations. Although the effective coupling between the $\mathrm{S}-\mathrm{T}_{+}$qubit states is timedependent, its form still allows to approximately reason in terms of LZSM physics.

With our formalism, we have compared results for different values of $\gamma_{0}$ and $\gamma_{2}$. Our findings suggest that LZSM spin interferometry is largely inhibited by charge dynamics, and thus charge coherence has to be treated on equal footing with spin coherence. We have indeed demonstrated that the visibility of the coherent oscillations of a spin qubit is sensitive to the time scale associated with charge induced spin dephasing. We have also shown that this interplay between charge and spin can prevent the observation of finite-time oscillations. Interestingly, this could lead to the development of an experimental protocol to measure the time scale associated with charge decoherence.

\section{ACKNOWLEDGEMENTS}

The authors are grateful to H. Lu and A. C. Gossard for assistance with device fabrication. H. R. and G. B. acknowledge funding from the DFG within SPP 1285 and SFB 767. Research at Princeton was supported by the Sloan and Packard Foundations and the National Science Foundation through the Princeton Center for Complex Materials, DMR-0819860 and CAREER award, DMR0846341.
* Current address: Department of Physics, University of Basel, Klingelbergstrasse 82, CH-4056 Basel, Switzerland

1 D. Loss and D. P. DiVincenzo, Phys. Rev. A 57, 120 (1998).

2 R. Hanson, L. P. Kouwenhoven, J. R. Petta, S. Tarucha, L. M. K. Vandersypen, Rev. Mod. Phys. 79, 1217 (2007).

${ }^{3}$ K. Ono, D. G. Austing, Y. Tokura, and S. Tarucha, Science 297, 1313 (2002).

4 M. Elzerman, R. Hanson, L. H. Willems van Beveren, B. Witkamp, L. M. K. Vandersypen, and L. P. Kouwenhoven, Nature 430, 431 (2004).

5 J. R. Petta, A. C. Johnson, J. M. Taylor, E. A. Laird, A. Yacoby, M. D. Lukin, C. M. Marcus, M. P. Hanson, and A. C. Gossard, Science 309, 2180 (2005).

${ }^{6}$ F. H. L. Koppens, C. Buizert, K. J. Tielrooij, I. T. Vink, K. C. Nowack, T. Meunier, L. P. Kouwenhoven, and L. M.
K. Vandersypen, Nature 442, 766 (2006).

7 K. C. Nowack, F. H. L. Koppens, Yu. V. Nazarov, and L. M. K. Vandersypen, Science 318, 1430 (2007).

${ }^{8}$ W. A. Coish and D. Loss, Phys. Rev. B 72, 125337 (2005).

9 J. M. Taylor, J. R. Petta, A. C. Johnson, A. Yacoby, C. M. Marcus, and M. D. Lukin, Phys. Rev. B 76, 035315 (2007).

10 S. Nadj-Perge, S. M. Frolov, E. P. A. M. Bakkers, and L. P. Kouwenhoven, Nature 468, 1084 (2010).

11 J. Levy, Phys. Rev. Lett. 89, 1147902 (2002).

12 I. van Weperen, B. D. Armstrong, E. A. Laird, J. Medford, C. M. Marcus, M. P. Hanson, and A. C. Gossard, Phys. Rev. Lett. 107, 030506 (2011).

13 M. D. Shulman, O. E. Dial, S. P. Harvey, H. Bluhm, V. Umansky, A. Yacoby, Science, 336, 202 (2012). 
14 S. Foletti, H. Bluhm, D. Mahalu, V. Umansky, and A. Yacoby, Nat. Phys. 5, 903 (2009).

15 A. V. Khaetskii, D. Loss, and L. Glazman, Phys. Rev. Lett. 88, 186802 (2002).

16 J. R. Petta, H. Lu, and A. C. Gossard, Science 327, 669 (2010).

17 H. Ribeiro, J. R. Petta, and G. Burkard, Phys. Rev. B 82, 115445 (2010).

18 H. Ribeiro, G. Burkard, J. R. Petta, H. Lu, and A. C. Gossard, Phys. Rev. Lett. 111, 086804 (2013).

19 L. D. Landau, Phys. Z. Sowjetunion 2, 46 (1932).

${ }^{20}$ C. Zener, Proc. R. Soc. A 137, 696 (1932).

21 E. C. G. Stückelberg, Helv. Phys. Acta 5, 369 (1932).

22 E. Majorana, Nuovo Cimento 9, 43 (1932).

23 S. N. Shevchenko, S. Ashhab, and F. Nori, Physics Reports 492, 1 (2010).

24 W. D. Oliver, Y. Yu, J. C. Lee, K. K. Berggren, L. S. Levitov, and T. P. Orlando, Science 310, 1653 (2005).

25 T. Calarco, A. Datta, P. Fedichev, E. Pazy, and P. Zoller, Phys. Rev. A 68, 012310 (2003).

${ }^{26}$ E. M. Gauger, S. C. Benjamin, A. Nazir, and B. W. Lovett, Phys. Rev. B 77, 115322 (2008).

27 J. H. Cole, A. D. Greentree, L. C. L. Hollenberg, and S. Das Sarma, Phys. Rev. B 77, 235418 (2008).

28 T. Hayashi, T. Fujisawa, H. D. Cheong, Y. H. Jeong, and Y. Hirayama, Phys. Rev. Lett. 91, 226804 (2003).

29 J. R. Petta, A. C. Johnson, C. M. Marcus, M. P. Hanson, and A. C. Gossard, Phys. Rev. Lett. 93, 186802 (2004).

30 I. I. Rabi, Phys. Rev. 51, 652 (1937).

${ }^{31}$ R. Hanson and D. D. Awschalom, Nature 453, 1043 (2008).

32 A. Abragam, Principles of Nuclear Magnetism, Oxford University Press, New York, (1961).

${ }^{33}$ N. Rosen and C. Zener, Phys. Rev. 40, 502 (1932).

${ }^{34}$ L. Allen and J. H. Eberly, Optical Resonance and TwoLevel Atoms, (Dover, New York, 1987).

${ }^{35}$ F. T. Hioe, Phys. Rev. A 30, 2100 (1984).

36 A. Bambini and P. R. Berman, Phys. Rev. A 23, 2496 (1981).

37 Y. N. Demkov and M. Kunike, Vestn. Leningr. Univ., Ser.Fiz.Khim. 16, 39 (1969).

38 F. T. Hioe and C. E. Carroll, Phys. Rev. A 32, 1541 (1985).

39 J. Zakrzewski, Phys. Rev. A 32, 3748 (1985).

40 Y. N. Demkov, JETP 45, 195 (1963) [Soviet. Phys. JETP 18, 138 (1964)].

41 E. E. Nikitin, Opt. Spektrosk. 13, 761 (1962) [Opt. Spectrosc. USSR 13, 431 (1962)].

42 C. E. Carroll and F. T. Hioe, J. Phys. A: Math. Gen. 19, 3579 (1986).

43 N. V. Vitanov, B. M. Garraway, Phys. Rev. A 53, 4288 (1996).

${ }^{44}$ M. Abramowitz and I. A. Stegun, Handbook of Mathematical Functions, Ch. 6, Dover (1970).

45 A. Erdélyi, W. Magnus, F. Oberhettinger, and F.G. Tricomi, Higher Transcendental Functions, Vol. II, Ch. 8, McGraw-Hill, New York, (1953).
${ }^{46}$ H. Bluhm, S. Foletti, I. Neder, M. Rudner, D. Mahalu, V. Umansky, and A. Yacoby, Nat. Phys. 7, 109 (2011).

47 S. A. Studenikin, G. C. Aers, G. Granger, L. Gaudreau, A. Kam, P. Zawadzki, Z. R. Wasilewski, and A. S. Sachrajda, Phys. Rev. Lett. 108, 226802 (2012).

${ }^{48}$ R. Hanson, L. M. K. Vandersypen, L. H. Willems van Beveren, J. M. Elzerman, I. T. Vink, and L. P. Kouwenhoven, Phys. Rev. B 70, 241304(R) (2004).

49 G. Burkard, D. Loss, and D. P. DiVincenzo, Phys. Rev. B 59, 2070 (1999).

50 Y. Kayanuma and S. Fukuchi, J. Phys. B 18, 4089 (1985).

51 T. Usuki, Phys. Rev. B 56, 13360 (1997).

52 J. Särkkä and A. Harju, New J. Phys. 13, 043010 (2011).

53 H. Ribeiro and G. Burkard, Phys. Rev. Lett. 102, 216802, (2009).

54 V. A. Abalmassov and F. Marquart, Phys. Rev. B 70, 075313 (2004).

55 X. Hu and S. Das Sarma, Phys. Rev. Lett. 96, 100501 (2006).

56 G. Lindblad, Commun. Math. Phys. 48, 119 (1976).

57 H. P. Breuer and F. Petruccione, Theory of open quantum systems, Oxford University Press, (2007).

58 A. V. Khaetskii and Y. V. Nazarov, Phys. Rev. B 64, 125316 (2001).

59 A. G. Redfield, IBM J. Res. Dev. 1, 19 (1957).

60 L. Chirolli and G. Burkard, Advances In Physics 57, 225 (2008).

61 L. DiCarlo, H. J. Lynch, A. C. Johnson, L. I. Childress, K. Crockett, C. M. Marcus, M. P. Hanson, and A. C. Gossard, Phys. Rev. Lett. 92, 226801 (2004).

62 P. Ao and J. Rammer, Phys. Rev. Lett. 62, 3004 (1989).

63 E. Shimshoni and Y. Gefen, Ann. Phys. 210, 16 (1991).

64 M. Wubs, K. Saito, S. Kohler, P. Hänggi, and Y. Kayanuma, Phys. Rev. Lett. 97, 200404 (2006).

65 K. Saito, M. Wubs, S. Kohler, Y. Kayanuma, and P. Hänggi, Phys. Rev. B 75, 214308 (2007).

66 P. Nalbach and M. Thorwart, Phys. Rev. Lett. 103, 220401 (2009).

67 P. P. Orth, A. Imambekov, K. Le Hur, Phys. Rev. A 82, 032118 (2010).

68 B. M. Maune, M. G. Borselli, B. Huang, T. D. Ladd, P. W. Deelman, K. S. Holabird, A. A. Kiselev, I. AlvaradoRodriguez, R. S. Ross, A. E. Schmitz, M. Sokolich, C. A. Watson, M. F. Gyure, and A. T. Hunter, Nature 481, 344 (2012)

69 D. E. Savage, M. G. Lagally, L. R. Schreiber, L. M. K. Vandersypen, Mark Friesen, Robert Joynt, S. N. Coppersmith, and M. A. Eriksson, Phys. Rev. Lett. 108, 046808 (2012).

70 K. Wang, C. Payette, Y. Dovzhenko, P. W. Deelman, J. R. Petta, arXiv:1304.2640 [Phys. Rev. Lett. (to be published)].

71 M. Pioro-Ladrière, T. Obata, Y. Tokura, Y.-S. Shin, T. Kubo, K. Yoshida, T. Taniyama, and S. Tarucha, Nat. Phys. 4, 776 (2008). 